\title{
The other side of the coin: Cognitive properties of nonreward
}

\author{
STEWART H. HULSE \\ Johns Hopkins University, Baltimore, Maryland
}

\begin{abstract}
In this comment, I examine Amsel's theory in the light of rats' reaction not to frustrative nonreward per se, but to the more complicated case in which frustrative nonreward is part of a rulebased sequence of reward quantities. The discussion goes beyond Amsel's emphasis on dispositional memory to consider cognitive and representational memory-the signaling properties of reinforcement and nonreinforcement. The strengths and weaknesses of Amsel's theory are discussed with the fresh perspective that this viewpoint provides, and some issues emerge that may be fruitful for further study.
\end{abstract}

Having followed much of Professor Amsel's work over the years, and having read his book (Amsel, 1992) and the précis of it (Amsel, 1994) which are the focus of this and the companion articles, I think it is pointless to dissect such a massive body of careful thinking and hard work with the aim of uncovering flaws. There may be some, but they are of relatively little importance when set against the enormous scope and impact of Amsel's science.

Instead, I would like to set Amsel's work in a larger context, a context in which I have had some things to say over the years. That context also deals with operations that produce frustrative nonreward in Amsel's terms, but it places emphasis not on the general, dispositional aspects of nonreward, but on the information or signaling value of reward and nonreward within a cognitive framework. What I want to say falls on the left side of Table 1 in Amsel's (1994) précis. Of course, this takes the discussion away from his theory - and for that reason he would be quite justified in ignoring or dismissing my thoughts altogether. Perhaps he will! But through my brief remarks, perhaps Amsel's work will gain some perspective that will, through contrast, further illuminate some of its unique properties. For example, one might ask what is lost in some larger sense through neglect of the frustration operations in the processes described at the left in Table 1? Or how do the processes specified by the two halves of the table interact?

I turn, then, to a brief discussion of some research, most of it from my laboratory over the years, that has stressed the stimulus information value of changes in reward quantities. All of the experiments involved plenty of contrasts among different quantities of reinforcement,

Preparation of this article was supported by National Science Foundation Grant BNS-8911046. Correspondence concerning the article should be addressed to S. H. Hulse, Department of Psychology, Johns Hopkins University, Baltimore, MD 21218 (e-mail: hulse@jhunix. hef.jhu.edu). including nonreinforcement - and hence, potentially, substantial amounts of frustration. But my focus was on what animals could learn about the stimulus relations among reinforcing events even as they dealt, no doubt, with the concomitant emotion they were experiencing. Instead of focusing so much on the within trial, emotional correlates of frustrative nonreward and the stimulus aftereffects and counterconditioning in the context associated with them, the work focused on the betweentrial, stimulus properties associated with reinforcement patterns more generally conceived-and on what they could lead the animal to learn about the flow of reinforcing events. The process was conceived to be more cognitive and perceptual than emotional.

\section{The Organization of Serial Sequences of Reinforcing Stimuli}

The inspiration for most of the work discussed here came from two seminal sources. The first was Lashley's (1951) paper on the problem of serial order in behavior, and the other was Simon and Kotovsky's (1963) paper on how the problem of serial order could be conceptualized for human learning in terms of rules. Lashley noted the deficiency of a pure stimulus-response associative chain model for describing how organisms learned that stimulus events followed one another, and Simon and Kotovsky described some principles (later expanded upon by Restle [e.g., 1970], among others) for the serial organization of stimulus events in memory. For example, Simon and Kotovsky described how people view a sequence of stimulus events (such as the numbers 1-35-7-9) and, when asked to supply the next stimulus in the sequence, typically provide the number 11 . In so doing on such a completion test, they exhibit the propensity to (1) abstract an organizing rule for the elements of the sequence, and (2) retrieve the rule and use it to generate the next element by extrapolation. It is important to note that these are both psychological principles that reside in the observer, not in the stimuli. A priori, the stimulus sequence in question might have been fol- 
lowed by any number. People just do not conceptualize the problem that way.

"Thinking ahead" in rat discrimination learning. The question was whether the foregoing ideas could be applied meaningfully to creatures other than humans. Hulse and Campbell (1975), in the first experiment on that problem, trained rats in a T-maze brightness discrimination. Correct choices were rewarded not with just one piece of food following an initial free-choice response, but instead with a sequence of reward quantities consisting of the initial food quantity plus four additional quantities to which the rats were forced over four further runs to the same side of the maze. That is, a trial consisted of an initial free-choice run to an arm in the T, followed by four forced runs to the same arm. Incorrect choices were treated similarly: If the rats chose the wrong side of the $\mathrm{T}$ on their initial free-choice run, nonreinforcement ensued, and that was followed by additional forced nonreinforced runs to the incorrect alternative. This procedure was designed to ensure that the rats had equal experience with the choice alternatives. The interrun interval within a trial was $30 \mathrm{sec}$, while the intertrial interval was $15-20 \mathrm{~min}$. This ensured that the runs of a trial were "chunked" together in time from trial to trial.

The sequences of reward quantities for correct choices all contained the same total amount of food, but the quantities were arranged from run to run in different orders. There were three critical groups in the experiment (plus some controls, which are not important here). A group trained with an increasing sequence (SEQ-I) had reward quantities increase from 0 (yes, the initial choice on a correct trial was not reinforced) through 1 , 3,7 , and 14 food pellets. A SEQ-D group had reward quantities decrease from 14 food pellets on the initial choice through 7,3,1, and 0 food pellets on subsequent runs. A random group received the food quantities in a haphazard order from one correct trial to another. The significant questions were, first, would the rats learn the discrimination under these conditions? Second, would they show differential sensitivity to the three sequences of food quantities for correct choices?

The discrimination was mastered under all conditions. Not surprisingly, perhaps, the SEQ-I group required more trials to reach a criterion than the others did, no doubt because of the confusion induced by the initial nonreinforcement of the free-choice run to the correct side (and, no doubt, frustration-induced incompatible responses that had to habituate over trials), but all groups learned. The running-time data for the free and forced runs to the correct side of the maze on correct choices are shown in Figure 1.

The significant fact to note from the figure is that the rats' running times reflected their anticipation of the successive quantities of food on the runs of a correct choice. Rats in the SEQ-I group were appropriately hesitant on the initial free-choice run; they ran fast thereafter (but no faster than the other groups). Rats in the SEQ-D group ran quickly at first, but then slowed in an-

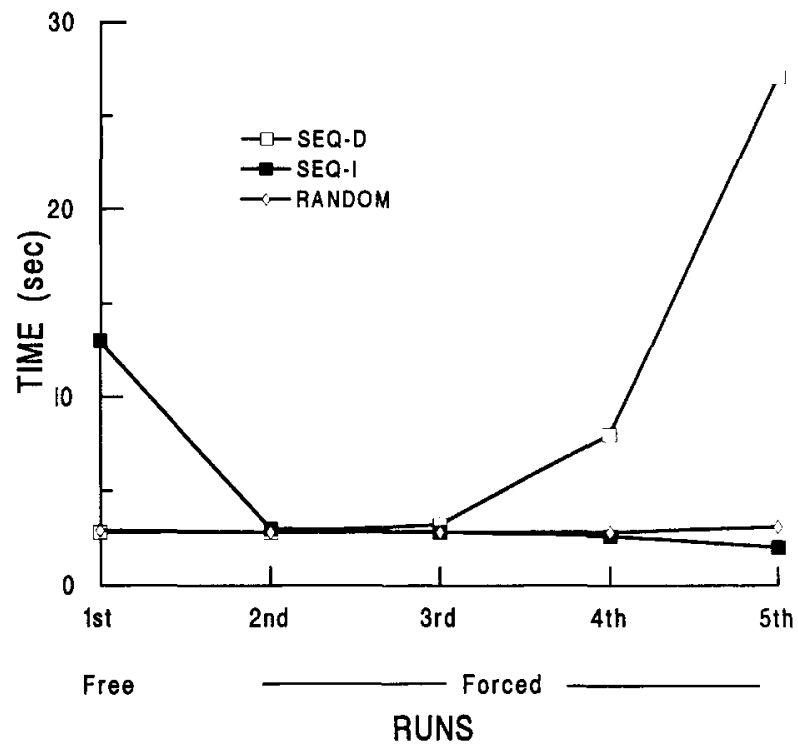

Figure 1. Response times to the correct arm of a T-maze on the initial free-choice run to that arm, and on the following four forced runs. The order in which $0,1,3,7$, and 14 pellets of food were obtained by the rats in that arm increased (SEQ-I), decreased (SEQ-D), or varied randomly. Data are from Hulse and Campbell (1975).

ticipation of nonreinforcement on the final forced run. Rats in the random group, unable to anticipate any given food quantity accurately, opted to run quickly on all trials.

What about data from nonreinforced runs to the incorrect side of the maze when rats made errors? First, it proved impossible to completely balance the number of free and forced runs to the correct and incorrect choices. The rats often refused to enter the wrong side of the maze on more than two or three of the successive forced runs that followed an incorrect choice-often they bit the experimenter and demonstrated other signs of nonreinforcement-produced frustration and aggression. So the procedure was modified to add just one nonreinforced run following the initial nonreinforced choice on an error trial. The relevant data appear in Figure 2.

For trials on which errors occurred, rats trained with predictably increasing or decreasing food sequences for correct responses were much slower to enter the wrong side of the maze - in comparison with rats for which reward sequences were haphazard following correct choices. This fact was true on the initial choice of the wrong side, but especially on the second forced run to the wrong side that ensued. These hesitancies to the wrong side following errors were characteristic only of the SEQ-I and SEQ-D groups - that is, for the groups in which reward sequences were arrayed in orderly fashion. The rats in the random group ran rapidly to the wrong side on both their free and forced choices to that side.

What are we to make of these data theoretically? One possibility - the one we adopted at the time - is that the rats abstracted the structure of the sequences of food quantities, encoded INCREASE, DECREASE, or RANDOM 


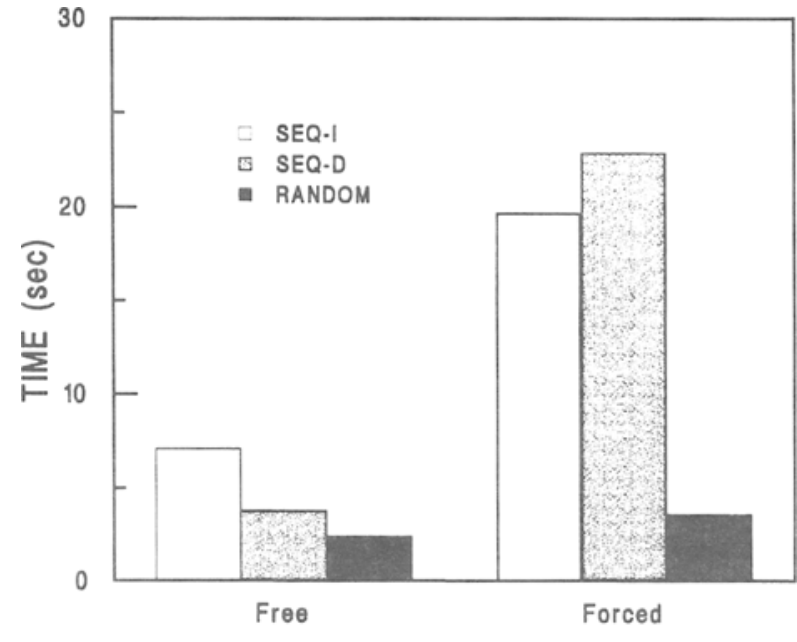

RUNS

Figure 2. Response times to the incorrect arm of a T-maze on the initial free-choice run to that arm, and on the following forced run, as a function of whether reward quantities increased (SEQ-I), decreased (SEQ-D), or varied randomly in the correct arm of the maze. Data are from Hulse and Campbell (1975).

rules describing that stimulus structure, and adjusted their running times in accordance with an application of the encoded rules (Hulse, 1978; Hulse \& Dorsky, 1977). This approach requires the one additional assumptiona reasonable one - that rats slow or speed their running in anticipation of large and small amounts of reward. The analysis is certainly based on principles to the left of center in Amsel's Table 1.

The extrapolation of serial patterns. The Hulse and Campbell (1975) data imply strongly that rats are very good at anticipating the next food quantity in a series of food quantities. They may even encode a rule describing the orderly changes in food quantities. One way to test that idea further is to train rats on different rulebased sequences and see whether they do, indeed, extrapolate the patterns on the basis of the rule when given an opportunity to do so.

Fountain and Hulse (1981) trained three groups of rats to run an alley for four different food quantities arranged in three sequences. With respect to the order of food quantities, one sequence, 14-7-3-1 food pellets, was strongly monotonic: each successive quantity was smaller than its predecessor. Another sequence, 14-5-5-1 food pellets, was weakly monotonic: each successive quantity was never larger than its predecessor but was sometimes equal. A third, 14-3-7-1 food pellets, was nonmonotonic: successive quantities were sometimes smaller and sometimes larger than their predecessors. The notion was that the three patterns, given that their organized structures were encoded, should vary in their implication of the size of a fifth food quantity should the rats be asked to make one more run and "fill in the blank," after the manner of the completion tests described by Simon and Kotovsky (1963). After initial training in which all groups responded with roughly the same rapid running times to all the quantities, the rats were given a transfer test in which they were returned to the runway after completing runs to the initial four food quantities as usual. The results on the very first trial of the transfer test are shown in Figure 3.

For all three conditions, the running times on the four quantities preceding the novel fifth run tended to slow, especially in anticipation of the final one-pellet quantity used in initial training. The main data of interest, however, are those for the critical fifth test run-to zero reinforcement (although, of course, the rats did not discover this fact on the first test run until they entered the goalbox). The figure shows clearly that the rats slowed their responding on the fifth run according to the extent to which the training patterns implied yet a smaller quantity of food following the fourth run. The implication was strongest in the structure of the strongly monotonic pattern, less so in the weakly monotonic pattern, and not implied at all in the nonmonotonic pattern. The rats behaved as if they encoded the structure of the patterns and accurately "predicted" the novel quantity through extrapolation of the rule. Although we have no way of knowing whether the rats were anticipating true nonreinforcement (and its associated frustration) on the test trial, the possibility is intriguing.

Other experiments. This is but a small sample of the research on serial patterns of food quantities. Other experiments have shown, among other things, that longer patterns, with more instances of a given rule, are more efficient in inducing rule encoding than shorter patterns are (Fountain, Evensen, \& Hulse, 1983). Furthermore, pattern learning is more efficient if pattern structures are parsed by spatial or temporal phrasing cues (Fountain, Henne, \& Hulse, 1984). More recently, Fountain (1990) has used brain stimulation as reinforcement and found

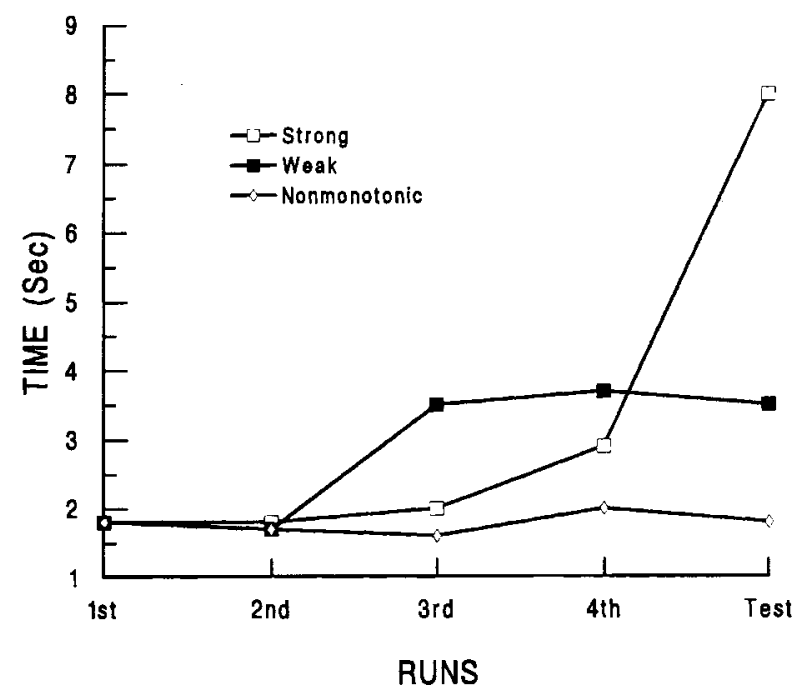

Figure 3. Response times on training runs and on the very first test run, as a function of whether reinforcement quantities on training runs decreased according to a strong monotonic rule, a weak monotonic rule, or a nonmonotonic rule. Data are from Fountain and Hulse (1981). 
hat rats are superbly skillful at learning very compliated rule-based sequences of reinforced and nonreinorced choices.

The role of frustrative nonreward in serial learning. 3ut what general role is frustrative nonreward playing in he foregoing situations, and what does it buy us theoetically? First, it cannot - alone, and as a general proess-account for the serial patterns of running times icross the groups following correct and incorrect choices $\mathrm{n}$ the Hulse and Campbell (1975) experiment, because tll the groups received exactly the same quantities of renforcement through the runs of a trial. Stimulus order $s$ paramount, and the effects of that are not captured efectively by principles of frustrative nonreward alone. jecond, in that experiment, frustrative nonreward as art of the overall discrimination process (see Amsel, 1994, Figure 2) no doubt accounted at least in part for he rats' eventual reluctance to enter the wrong alley. However, it is difficult to see how it would explain an inrreased reluctance to run on the forced return to the vrong alley only in the rats experiencing rule-based paterns of reinforcement and nonreinforcement in the corect, reinforced alternative of the T-maze (cf. Figure 2). Iranted that perseverative effects based on the generalzation of conditioned persistence from one alternative o the other might occur (see, especially, chapter 4 of Frustration Theory, and Amsel's extensive discussion in is précis of the transfer of persistence effects across zonditions). But if so, the generalized effects are overaid by a process that reflects the ordering of stimulus jequences associated with the alternative, correct choice. And that seems better accounted for by generalization zased on a process such as the rule-based approach outlined above.

It is hard to know how Amsel would deal with the extrapolation of food quantities in the Fountain and Hulse (1981) experiment. In that case, the rats were apparently anticipating reduced (zero?) reinforcement based on rule structure. Were they also anticipating primary frustration under those circumstances, even though they had never experienced it directly?

So, to sum up, these data do not seem to be explained adequately by an appeal to frustrative nonreward alone, although, as I have noted, frustrative nonreinforcement was no doubt part of the overall learning situation. If Professor Amsel will permit, one may imagine a rat, hungry and under pressure, who nevertheless comes to survey and control the discrimination situation coolly - encoding and utilizing the many regularities with respect to reinforcing stimuli that the situation affords. That rat is clearly following the principles outlined at the left of Table 2 in Amsel's précis, while at the same time dealing, no doubt, with correlates of the principles on the right.

\section{Serial Sequences and the Brain}

Observations like those just described invite questions about the brain structures that underlie them. To my knowledge, little or no work has been done on this issue in the context of serial sequences of reinforcement. One of the few, if not the only, experiments of which I am aware is an experiment done by Olton, Shapiro, and Hulse (1984), in which rats with fimbria-fornix lesions were impaired in their ability to track a series of decreasing quantities of food placed in the arms of a radial arm maze. Given the stress that Amsel places on the hippocampus in mediating the effects of partial reinforcement on behavior, it remains important to do further experiments examining the function of that structure in this regard.

\section{Questions}

I close by identifying an issue that may, perhaps, stimulate some discussion. It is possible to think of nonreinforcement and any particular finite quantity of reinforcement as special cases of a larger set of eventsnamely, variable quantities of reinforcement. This implies in turn that the functions that Amsel ascribes to the hippocampus and the behavioral inhibition system (BIS) in mediating the effects of reinforcement and nonreinforcement must apply to this larger class of events. If that is correct, the hippocampus (and the BIS) must be more finely tuned to reinforcing events than to simple reinforcement and nonreinforcement. If so, what implications does that have for understanding the neural underpinnings of persistence and the other effects ascribed to frustrative nonreinforcement? What implications does this have, similarly, if the variable quantities of reinforcement are arrayed in some rule-based organized series? And how might this modify Amsel's conceptualization of the development of the "paradoxical" effects of reinforcement and nonreinforcement-the development, for example, of the partial reinforcement effect in ontogeny?

It would be fun and, no doubt, fruitful to apply Amsel's "six steps" (Amsel, 1994, Table 3) to the issues raised by the foregoing questions. This is not the place to discuss the application of the steps to the issues in detail. But it would be interesting, for example, to know more about the neurophysiological control of the effects of varied (and ordered) patterns of reinforcement, and a good place to start would be to search for their appearance in development. Is the ability of a rat to "track" a sequence of reward quantities related to the ontological emergence of sensitivity to reward and nonreward per se? Offhand, that seems a reasonable conjecture worthy of study.

Of course, all these issues and questions become important largely through the theoretical context in which they exist. We all owe Professor Amsel great credit for creating that setting and developing it so skillfully.

\section{REFERENCES}

Amser, A. (1992). Frustration theory: An analysis of dispositioned learning and memory. New York: Cambridge University Press.

AMSEl, A. (1994). Précis of Frustration theory: An analysis of dispositional learning and memory. Psychonomic Bulletin \& Review, 1 , 280-296. 
Fountain, S. B. (1990). Rule abstraction, item memory, and chunking in serial-pattern tracking. Journal of Experimental Psychology: Animal Behavior Processes, 6, 96-105.

Fountain, S. B., Evensen, J. C., \& Hulse, S. H. (1983). Formal structure and pattern length in serial pattern learning by rats. Animal Learning \& Behavior, 11, 186-192.

Fountain, S. B., Henne, D. R., \& Hulse, S. H. (1984). Phrasing cues and hierarchical organization in serial pattern learning by rats. Journal of Experimental Psychology: Animal Behavior Processes, 10 $30-45$.

Fountain, S. B., \& Hulse, S. H. ( 1981). Extrapolation of serial stimulus patterns by rats. Animal Learning \& Behavior, 9, 381-384.

HuLSE, S. H. (1978). Cognitive structure and serial pattern learning by animals. In S. H. Hulse, H. Fowler, \& W. K. Honig (Eds.), Cognitive processes in animal behavior (pp. 311-340). Hillsdale, NJ; Erlbaum.

HulsE, S. H., \& CAMPBELL, C. E. (1975). "Thinking ahead" in rat discrimination learning. Animal Learning \& Behavior, 3, 305-311.
Hulse, S. H., \& Dorsky, N. P. (1977). Structural complexity as a determinant of serial pattern learning. Learning \& Motivation, 8, 488506.

LASHLEY, K. S. (1951). The problem of serial order in behavior. In L. A. Jeffress (Ed.), Cerebral mechanisms in behavior (pp. 112146). New York: Wiley.

Olton, D. S., Shapiro, M. L., \& Hulse, S. H. (1984). Working memory and serial patterns. In H. L. Roitblat, T. G. Bever, \& H. S Terrace (Eds.), Animal cognition (pp. 171-182). Hillsdale, NJ: Erlbaum.

RestLE, F. (1970). Theory of serial pattern learning: Structural trees. Psychological Review, 77, 481-495.

Simon, H. A., \& KotovsKy, K. (1963). Human acquisition of concepts for sequential patterns. Psychological Review, 70, 534-546.

(Manuscript received January 17, 1994; revision accepted for publication March 28, 1994.) 